le portiQue $\begin{array}{ll}\text { Le Portique } \\ \text { Revue de philosor }\end{array}$

13-14 | 2004

Foucault : usages et actualités

\title{
Paul Ricœur et Michel Foucault
}

\section{Rose Goetz}

\section{OpenEdition}

Journals

Édition électronique

URL : http://journals.openedition.org/leportique/639

DOI : 10.4000/leportique.639

ISSN : $1777-5280$

Éditeur

Association "Les Amis du Portique"

Édition imprimée

Date de publication : 1 septembre 2004

ISSN : 1283-8594

Référence électronique

Rose Goetz, "Paul Ricœur et Michel Foucault », Le Portique [En ligne], 13-14 | 2004, mis en ligne le 15 juin 2007, consulté le 26 mars 2021. URL : http://journals.openedition.org/leportique/639; DOI : https://doi.org/10.4000/leportique.639

Ce document a été généré automatiquement le 26 mars 2021.

Tous droits réservés 


\section{Paul Ricœur et Michel Foucault}

\section{Rose Goetz}

1 Ricœur et Foucault ? Peut-on associer aujourd'hui - et, si oui, de quelle façon - deux pensées restées longtemps étrangères l'une à l'autre, oscillant, l'une à l'égard de l'autre, entre ignorance et hostilité ? Le lecteur des ouvrages publiés par les deux philosophes au cours des années 1960 et 1970 ne pouvait guère imaginer entre eux de rapports autres que d'indifférence ou d'antagonisme latent. Ce n'est donc pas sans surprise que ce même lecteur découvre, dans plusieurs textes postérieurs à la mort de Foucault, l'hommage appuyé que lui rend Ricœur.

Dès 1985, dans Temps et récit III, on note une prise en compte de L'Archéologie du savoirtrès critique, il est vrai - qui érige Foucault en interlocuteur reconnu par Ricœur alors soucieux de réfuter la thèse selon laquelle la continuité de la mémoire serait une illusion idéaliste. Concernant la nécessité même d'une archéologie du savoir opposée à l'histoire des idées, ce dialogue reprendra en 2000, dans la Mémoire, l'histoire, l'oubli, où Foucault apparait, avec Michel de Certeau et Norbert Elias, en " maître de rigueur " pour l'historiographie contemporaine. Mais la discussion engagée en 1985, à propos du concept de "formation discursive ", se déploie encore largement dans le sillage de la réprobation que les Mots et les Choses avait inspirée à Ricœur en 1966 et qu'il évoque, en 1995, dans La Critique et la Conviction: "L'idée que l'homme est une invention récente me paraissait tout simplement fabuleuse. Je pense par exemple à l'“Ode à l'homme" dans l'Antigone de Sophocle [...]. Comment oublier aussi le souci stoïcien, la maîtrise des désirs et des passions, auxquels précisément Foucault revient à bien des égards dans ses derniers textes, que j'admire beaucoup, L'Usage des plaisirs et Le Souci de soi. Mais justement, c'est presque une autre philosophie que celle qu'il avait développée dans les Mots et les Choses. J'étais très réservé à l'égard de ce livre. L'idée des épistémè qui se remplacent les unes les autres avec des transitions aléatoires, non seulement me semblait inintelligible, mais surtout je trouvais qu'elle ne reposait pas sur une richesse de contenu assez grande pour chacune de ces épistémè. Foucault me paraissait faire à chaque fois un prélèvement trop limité pour être probant ${ }^{1}$. Le passage de cette "réserve" envers le premier Foucault à l'admiration éprouvée pour le dernier est porté au compte d'une mutation de sa philosophie: "C'est dans la mesure où Foucault s'est 
éloigné de lui-même, avec ses deux derniers livres, que je me suis senti plus proche de lui" » ${ }^{2}$.

3 Dans un entretien paru dans le quotidien La Croix le 26 février 2003, et reproduit dans le récent Cahier de l'Herne qui lui est consacré, Ricœur réaffirme superlativement son admiration pour Foucault, associé cette fois à Deleuze. Évoquant une période où luimême n'était pas encore tenu pour un philosophe important, mais où, enseignant heureux et estimé, il ne souffrait pas de ce manque de reconnaissance, il précise : «Je ne ressentais donc pas de ne pas être jugé l'égal de Deleuze, de Foucault, pour nommer les deux penseurs que, par ailleurs, j'ai le plus admirés » ${ }^{3}$. Peut-être antérieure à la lecture de L'Usage des plaisirs et du Souci de soi, cette admiration de Ricœur pour Foucault s'est liée, en 1984, à un sentiment de proximité de pensée que n'élucide guère le passage de La Critique et la Conviction, que j'ai commencé à citer: "C'est dans la mesure où Foucault s'est éloigné de lui-même, avec ses deux derniers livres, que je me suis senti plus proche de lui; mais sans avoir l'occasion de le lui dire. C'est une rencontre qui n'a pas eu lieu. Certainement que lui n'en attendait rien, et moi j'étais sur des chemins où je le rencontrais peu, sinon par des intersections très ponctuelles " ${ }^{4}$. $\mathrm{Ce}$ rapprochement s'étant manifestement produit à l'occasion de l'exploration par Foucault du souci de soi dans l'Antiquité gréco-romaine, le lecteur est tenté d'en rechercher quelques signes dans les ouvrages de Ricœur portant sur le soi, essentiellement Soi-même comme un autre (1990) et Parcours de la Reconnaissance (2004).

Aucune référence ne le guidera dans sa recherche. Foucault apparaît bien une fois, dans la Préface de Soi-même comme un autre, mais en simple caution de la justesse d'une observation grammaticale attestant que le pronom « soi » désigne le réfléchi de tous les pronoms personnels, et même des pronoms impersonnels tels que "chacun", "quiconque ", «on ", de la même manière que le pronom «se » rapporté à des verbes au mode infinitif : «C'est cette valeur de réfléchi omnipersonnel qui est préservée dans l'emploi du "soi" dans la fonction de complément du nom ; le "souci de soi" - selon le titre magnifique de Michel Foucault ${ }^{5}$ (éloge peut-être excessif, le titre de Foucault n'étant que la traduction de l'epimeleia heautou des Grecs). Sur ce que Foucault entend par «souci de soi ", Ricœur ne dit mot: silence moins insolite qu'il peut sembler à première vue, l'analyse de la problématisation des comportements sexuels dans les textes grecs et latins des deux premiers siècles de notre ère n'entrant pas dans le cadre des études qui composent son livre.

5 C'est bien pourtant leur commun souci du souci de soi, source de leurs interrogations concernant le sujet et la subjectivité, l'exégèse de soi, la constitution de soi par soi, qui, pour le lecteur des deux philosophes, les relie l'un à l'autre. Ce lecteur doit, certes, se défier de la trompeuse parenté des thèmes traités ici et là. Intégrés dans des problématiques hétérogènes, ils y prennent des sens différents. Un parcours hâtif des derniers ouvrages de Foucault et de ses Cours au Collège de France dans les années qui en précédent de peu la parution (ne s'y aventure-t-il pas sur le terrain de l'herméneutique ?) pourrait faire croire que c'est lui qui s'est alors rapproché de Ricœur. Il n'en est rien sans doute. Les voies qui les conduisent à l'objet de leur commune préoccupation ne se croisent pas (sauf en quelques "intersections très ponctuelles»?). C'est donc à emprunter patiemment ces voies distinctes que doit s'astreindre le lecteur désireux de confronter ces deux approches du sujet et d'en dégager, à ses risques et périls, ce qui a pu motiver chez Ricœur le sentiment d'un rapprochement avec le dernier Foucault. 
6 Mais on peut adopter une autre attitude envers leurs œuvres respectives: les considérer comme des «boîtes à outils ", en extraire des instruments aptes, en certains cas, à s'agencer les uns aux autres, s'en servir à des fins théoriques et pratiques que ne concerne plus le respect scrupuleux des problématiques. Se laissent ainsi configurer pour le lecteur - plusieurs terrains de rencontre entre Ricœur et Foucault.

7 Je relèverais d'abord une conjonction fructueuse des regards neufs que leur souci du souci de soi leur fait porter sur l'Antiquité grecque. Ils s'intéressent rarement aux mêmes auteurs et, quand ils le font, ils n'en donnent pas la même lecture, ne mettent pas l'accent sur les mêmes concepts. Mais l'attention qu'ils portent à l'usage du pronom réfléchi hautô/heauto dans les textes les mène à ouvrir ensemble un vaste champ de réflexion sur le rapport de soi à soi dont il est question dans ces textes. Pour en prendre la mesure, le lecteur peut associer avec bonheur l'analyse par Foucault de l'Alcibiade de Platon ${ }^{6}$ et les analyses par Ricœur de maints chapitres de l'Éthique à Nicomaque d'Aristote, association non exclusive de bien d'autres possibles. L'un et l'autre, chacun à sa manière, montrent que si les Grecs ont ignoré nos concepts de volonté, de liberté, de conscience de soi, tels que les ont spéculativement élaborés les Modernes, se maintiennent, ou se redécouvrent, entre eux et nous, de fortes accointances au plan de la conception des mœurs, des expériences et des comportements, au plan d'une sagesse (ou d'une quête de sagesse) autoréflexive d'ordre pratique. Au-delà de son intérêt historico-philosophique, qui n'est pas mince, ce double éclairage projeté sur des textes anciens a une portée éthique et politique actuelle. On peut en tirer de précieuses leçons de vie au sein des situations qui sont les nôtres, non celles de leurs auteurs.

Le terrain apparemment le plus favorable à une rencontre entre Ricœur et Foucault est précisément celui de l'éthique, que tous deux distinguent de la morale, entendue comme code de prescriptions et d'interdictions imposées au sujet, tout en reconnaissant entre elles des liens réels, impossibles à rompre. Au troisième paragraphe de l'Introduction à l'Usage des plaisirs, Foucault oppose les morales «orientées vers le code», dans lesquelles règnent les règles et les interdits, et les morales " orientées vers l'éthique » dont « l'élément fort et dynamique est à chercher du côté des formes de subjectivation et des pratiques de soi ${ }^{7}$. Or ce qui, dans l'éthique, caractérise, entre autres traits, l'action d'un sujet est sa téléologie : elle vise à l'accomplissement de soi, à ce que, dans le Souci de soi, Foucault appelle la «culture de soi " menant à l'«allégresse " qu'évoque Sénèque dans l'une des Lettres à Lucilius : "Tourne ton regard vers le bien véritable; sois heureux de ton propre fonds (de tuo). Mais ce fonds, quel est-il ? Toi-même et la meilleure partie de toi ${ }^{8}$. Il paraît ainsi s'approcher de la distinction qu'établira Ricœur entre l'éthique, définie par sa visée téléologique et eudémoniste, et la morale, conçue comme déontologique, celle-ci entretenant avec celle-là un rapport qui est à la fois de subordination et de complémentarité. Mais la différence est grande entre les deux perspectives. Pour Ricœur, les normes morales ne se caractérisent pas seulement par un effet de contrainte, mais aussi par leur légitime prétention à l'universalité : l'héritage kantien est assumé. Si l'éthique, animée par le souhait de la "vie bonne " (héritage aristotélicien), est plus fondamentale que la morale, elle requiert la morale « dès lors que le souhait de la vie bonne rencontre la violence sous toutes ses formes " ${ }^{9}$. En outre, il est des cas aporétiques, tels les conflits de devoirs, où ni l'éthique ni la morale, comprises stricto sensu, ne suffisent à guider l'action. Aussi Ricœur considère-t-il un troisième niveau de l'éthique comprise lato sensu: celui de la sagesse pratique, 
inspirant, dans chaque cas difficile, un jugement moral en situation. Au plan de la désignation de soi par le sujet agissant, à la visée éthique de la vie bonne et heureuse correspond l'estime de soi ; au moment déontologique correspond le respect de soi, qui est l'aspect que revêt l'estime de soi sous le régime de la norme ; au troisième moment, «lorsqu'aucune norme certaine n'offre plus de guide sûr pour l'exercice hic et nunc du respect ", «l'estime de soi n'apparait pas seulement comme la source mais comme le recours du respect $»{ }^{10}$.

9 Les rapports qu'établit Ricœur entre éthique et morale sont donc sensiblement différents de ceux établis par Foucault. Mais il arrive que leurs analyses se fassent écho : il y a plus qu'une simple analogie entre la jouissance de soi, le plaisir qu'on prend à soi-même, le bonheur que l'on tire de son propre fonds, selon l'expression de Sénèque relevée par Foucault, et l'estime de soi que procure la visée du bien-vivre, pensée par Ricœur dans le sillage d'Aristote, et qui pourrait l'être aussi bien dans celui de Spinoza et de l'acquiescentia in se ipso (référence est faite à l'Éthique dans la $10^{\circ}$ étude de Soi-même comme un autre). Toutefois, le bénéfice principal que le lecteur peut retirer de la lecture conjointe des textes de Ricœur et de Foucault sur l'éthique est de concevoir celle-ci à la fois comme visée d'une vie bonne et comme visée d'une vie belle. Aux analyses par Ricœur de la première visée se laissent articuler les réflexions de Foucault sur les « arts de l'existence ", sur les techniques par lesquelles on fait de sa vie " une œuvre qui porte certaines valeurs esthétiques et réponde à certains critères de style ${ }^{11}$.

Ces arts de l'existence sont des pratiques par lesquelles les hommes cherchent à se transformer eux-mêmes, à se modifier dans leur être singulier, à éprouver ce dont ils sont capables. La thématique de l'«homme capable», centrale dans les principaux ouvrages de Ricœur depuis 1990, est l'un de ces carrefours où il pourrait parfois croiser Foucault. Mais, comme précédemment, on se heurte ici à l'hétérogénéité des questionnements. La question de l'homme capable surgit chez Ricœur au sein d'une herméneutique du soi qui n'est pas l'herméneutique du sujet de Foucault: elle se déploie dans le cadre d'une philosophie réflexive ignorée des Anciens ${ }^{12}$. L'herméneutique du soi est le lieu d'articulation de trois problématiques: 1) Le détour de la réflexion par l'analyse (au sens de la philosophie analytique) imposé par l'usage $\mathrm{du}$ « se » et du «soi » en cas obliques. Le soi n'est pas l'ego. On ne peut l'approcher qu'indirectement. 2) La dialectique entre l'identité - idem et l'identité - ipse, entre la mêmeté et l'ipséité : l'ipséité se détermine par la voie de son contraste avec la mêmeté. 3) La dialectique de l'ipséité et de l'altérité : altérité de l'autre que soi et de l'autre en soi. L'interprétation de soi par soi passe par cette triple médiation. Le soi est la réponse à la question qui ? Soi-même comme un autre inventorie une série de figures du qui : qui peut parler? qui peut agir? qui peut se raconter? qui peut s'imputer ses propres actes? Or, en de certaines limites et sous certaines conditions, je peux parler, je peux agir, je peux me raconter, je peux me tenir responsable de mes actes. Aux yeux de Ricœur, comme il le rappelle dans Parcours de la reconnaissance, la suite des figures les plus remarquables du «je peux» constitue "l'épine dorsale d'une analyse réflexive où le «je peux», considéré dans la variété de ses emplois, donnerait sa plus grande amplitude à l'idée d'action une première fois thématisée par les Grecs ${ }^{13}$. Aux antipodes de cette herméneutique du soi - qui doit sa première et décisive impulsion à la philosophie du cogito de Descartes et à la théorie de la réflexion de Locke - certaines thèses de Foucault s'y laissent, semble-t-il, raccorder, concernant le travail de transformation de soi et de conversion à soi sur lequel portent nombre de textes anciens, stoïciens notamment. Parmi les procédures de ce travail, les « récits de soi » 
qu'analyse Foucault peuvent également être lus à la lumière de la théorie de l'« identité narrative » élaborée par Ricœur ${ }^{14}$.

11 Je me borne à l'indication de ces quelques points de jonction possible entre les deux pensées du soi. Ils n'émergent qu'à traiter les textes comme des "boîtes à outils". Foucault a préconisé cet usage des siens. Ricœur, non. Mais ne donne-t-il pas maintes fois lui-même l'exemple d'une telle pratique, usant du droit qui est celui de «tout lecteur devant qui tous les livres sont simultanément ouverts ${ }^{15}$.

\section{NOTES}

1.. Paul RICefur, La Critique et la Conviction, Paris, Calmann-Lévy, 1997, p. 122-123.

2.. Ibid., p. 123.

3.. Ricœur, Paris, Éditions de l'Herne, 2004, p. 17.

4.. La Critique et la Conviction, op. cit., p. 123.

5.. Soi-même comme un autre, Paris, Seuil, 1990, p. 12.

6.. Voir L'Herméneutique du sujet, Cours au Collège de France, 1981-1982, Paris, Seuil/ Gallimard, 2001.

7.. Michel foucault, L'Usage des plaisirs, Paris, Gallimard, 1984, p. 37.

8.. Michel foucault, Le Souci de soi, Paris, Gallimard, 1984, p. 84.

9.. Paul RIC๕Un, Réflexion faite. Autobiographie intellectuelle, Paris, Esprit, 1995, p. 80.

10.. Soi-même comme un autre, p.201.

11.. L'Usage des plaisirs, p. 16-17.

12.. Voir Parcours de la reconnaissance, Paris, Stock, 2004, p. 137.

13.. Ibid., p.139.

14.. Voir Michel foucault, «L'écriture de soi », Corps écrit, $\mathrm{n}^{\circ} 5$ : L'autoportrait, février 1983. Paul RICGUR, Soi-même comme un autre, $5^{\mathrm{e}}$ et $6^{\mathrm{e}}$ étude; Parcours de la reconnaissance, 2 étude, II, 3.

15.. Paul RICœUR, La Mémoire, l'histoire, l'oubli, Paris, Seuil, 2000, p. III.

\section{RÉSUMÉS}

Très critique envers Les Mots et les Choses, Ricœur dit son admiration pour L'Usage des plaisirs et Le Souci de soi et le rapprochement de sa pensée avec celle du dernier Foucault, laissant au lecteur la tâche, difficile, d'en trouver éventuellement les signes dans Soi-même comme un autre et dans d'autres écrits portant sur la question du soi.

Mais, sans jamais méconnaître la différence entre les problématiques des deux philosophes, le lecteur peut aussi, en vue d'élaborer sa propre compréhension de la question, traiter leurs textes 
comme des «boîtes à outils» et en extraire quelques instruments propres à s'articuler fructueusement les uns aux autres.

Quite critical towards Les Mots et les Choses, Ricœur tells of his admiration of L'Usage des plaisirs and le Souci de soi and exposes the connections of his thought with that of Foucault's latest, leaving the reader with the difficult task to possibly find traces in Soi-même comme un autre and in other texts on the question of self.

Keeping in mind the difference between the two philosophers' problematics, so as to elaborate their own understanding of the question, the readers can also consider their texts as a toolbox and extract some instruments that fruitfully combine together. 